\title{
ALTERAÇÕES MORFOLÓGICAS EM CÉREBROS DE PESSOAS DIAGNOSTICADAS COM DEPRESSÃO: REVISÃO INTEGRATIVA DA LITERATURA
}

\section{MORPHOLOGICAL CHANGES IN BRAINS OF PEOPLE DIAGNOSED WITH DEPRESSION: INTEGRATIVE LITERATURE REVIEW}

Ademilson José Bernardineli ${ }^{1}$, Claudia Regina Marchiori Antunes Araújo², Larissa Renata de Oliveira Bianchi ${ }^{1}$.

1Universidade Estadual de Maringá, Maringá, PR, Brasil.

${ }^{2}$ Instituto Adventista Paranaense, Ivatuba, PR, Brasil.

*adejb@hotmail.com

Recebido em: 27/06/2019; Aceito em: 08/10/2020.

\section{RESUMO}

Segundo a Organização mundial da saúde (OMS), a depressão é um transtorno comum que tem crescido exponencialmente em todo o mundo, com uma estimativa de mais de 300 milhões de pessoas acometidas por esta condição, 0 que a torna um problema de saúde pública. Dentre as consequências, destacase o comprometimento das atividades cotidianas e sociais dos indivíduos em todas as faixas etárias, podendo ocasionar desfechos trágicos e risco de vida. $O$ presente estudo tem como objetivo estudar as alterações morfológicas em cérebros de pessoas diagnosticadas com depressão. Para tanto, foi realizada uma revisão integrativa da literatura nas bases de dados Scielo e Medline. Após leitura criteriosa, foram selecionados cinco artigos que responderam aos critérios de inclusão. A análise dos artigos mostrou que cérebros de pessoas diagnosticadas com depressão apresentam alterações visíveis em exames de neuroimagens, como a ressonância magnética. As alterações identificadas foram volumes subcorticais menores, volumes ventriculares maiores, menor volume da substância cinzenta do córtex orbitofrontal, anormalidade no córtex pré-frontal, perda de diferenciação entre substância branca e cinzenta e envolvimento das estruturas límbicas. O estudo evidenciou, portanto, que os exames de neuroimagem podem apresentar-se como um recurso importante para a obtenção de um diagnóstico mais rápido e preciso dessa patologia.

Palavras-chave: Anatomia. Cérebro. Depressão. Diagnóstico. Neuroimagem.

\section{ABSTRACT}

According to the World Health Organization (WHO), depression is a common disorder that has grown exponentially worldwide, with an estimated over 300 million people affected by this condition, which makes it a public health problem. Among the consequences, we highlight the impairment of daily and social activities of individuals in all age groups, which can cause tragic outcomes and risk of life. The present study aims to study the morphological changes in the brains of people diagnosed with depression. For that, an integrative literature 
review was carried out in the data bases Scielo and Medline. After careful reading, five articles were selected that met the inclusion criteria. The analysis showed that the brains of people diagnosed with depression show visible changes in neuroimaging tests, such as magnetic resonance imaging. The identified changes weresmaller subcortical volumes, larger ventricular volumes, lower volume of gray matter in the orbitofrontal cortex, abnormality in the prefrontal cortex, loss of differentiation between white and gray matter and involvement of limbic structures. The study showed, therefore, that neuroimaging exams can present themselves as an important resource for obtaining a faster and more accurate diagnosis of this pathology.

Keywords: Anatomy. Brain. Depression. Diagnosis. Neuroimaging.

\section{INTRODUÇÃO}

A depressão é reconhecidamente um problema de saúde pública, e evidencia-se pelo comprometimento das atividades cotidianas do indivíduo, principalmente nas relações sociais (STOPA et al., 2015). Existem diversas abordagens no estudo da depressão, uma vez que esta pode ser decorrente de fatores genéticos, psicológicos, familiares e sociais. Essa psicopatologia caracteriza-se como um conjunto de transtornos, sendo sistematizados como "transtorno de humor" pelo Manual Diagnóstico e Estatístico de Transtornos mentais (DSM-IV) e "transtornos afetivos" pela Classificação Internacional de Doenças (CID 10).

Pessoas deprimidas buscam com frequência Unidades Básicas de Saúde, com queixas de diversos sintomas, porém, muitas vezes, não são diagnosticados como tal. Um Relatório da Organização Mundial de Saúde (OMS) aponta que o número de casos de depressão aumentou 18\% entre 2005 e 2015, com 322 milhões de pessoas em todo o mundo, sendo a maioria mulheres. No Brasil, a depressão atinge 11,5 milhões de pessoas (5,8\% da população) (WHO, 2017). De acordo com achados de Lopes et al. (2016), a maioria dos brasileiros com sintomas depressivos clinicamente relevantes $(78,8 \%)$, não recebem nenhum tipo de tratamento.

O diagnóstico da depressão é comumente estabelecido com base em correlações clínicas, através de anamnese, avaliação do histórico familiar e aplicação de algum instrumento ou protocolo que vise aproximar os sintomas apresentados pelo paciente ao diagnóstico. Diversos instrumentos de Screening para detecção de depressão na população foram desenvolvidos, como: World Health Organization Quality of Life (WHOQOL-bref), Mental Health Index5 (MHI5), Centers for Epidemiologic Studies - De-pression (CES-D), sendo o mais utilizado deles o Patient Health Questionnaire (PHQ-9), desenvolvido pela Associação Psiquiátrica Americana (APA) (SANTOS et al., 2013). Esses instrumentos têm sido avaliados como um avanço na detecção de possíveis casos de depressão na população, dada sua sensibilidade e confiabilidade.

Estudos como os de Coffey et al. (1998), sugeriram que cérebros de pessoas deprimidas apresentam alterações morfofuncionais que, quando submetidas à exames de neuroimagem, mostram maior frequência de hipersinais, e esclarecem que os estudos neuropsicológicos associados à 
neuroimagem trouxeram considerável avanço para o entendimento dos correlatos neuroanatomofuncionais dos transtornos psiquiátricos.

Em relação aos exames de imagem, distinguem-se dois tipos de acordo com seus objetivos: a Tomografia Computadorizada (TC) e a Ressonância Magnética (RM), que avaliam alterações estruturais do cérebro; a Ressonância Magnética Funcional (RMF), a Tomografia por Emissão de Pósitrons (PET), e a Tomografia por Emissão de Fóton Único (SPECT) que geram imagens que descrevem o estado funcional e metabólico do cérebro como um todo, bem como de regiões específicas, permitindo a investigação sem a utilização de métodos invasivos (WEIGHT; BIGLER, 1998).

Neste sentido, exames de neuroimagem apontam uma proposta metodológica clínica para a obtenção de um diagnóstico mais preciso dessas doenças, reduzindo erros e riscos de tratamentos associados à medicação inadequada. Sendo assim, este estudo pretende compreender, através da análise de artigos científicos, as principais alterações morfológicas observadas em cérebros de pessoas diagnosticadas com depressão.

\section{METODOLOGIA}

O estudo foi realizado através de uma Revisão Integrativa da Literatura. A metodologia utilizada congrega os resultados de pesquisas primárias sobre 0 tema escolhido, objetivando a síntese e análise desses dados de forma a desenvolver um esclarecimento mais amplo sobre o fato específico, e fornecendo um maior conhecimento do assunto discutido (COOPER, 1998).

A coleta de dados ocorreu em novembro de 2018, e foram incluídos os artigos publicados nos últimos cinco anos nas bases de dados Scielo e Medline, utilizando os descritores (DECs): anatomia, cérebro, depressão, neuroimagem e diagnóstico. Foram incluídos artigos que abordaram o diagnóstico da depressão relacionado ao desenvolvimento morfológico do cérebro e à neuroimagem. Foram excluídos os artigos que não responderam ao objetivo deste trabalho, que não estavam disponíveis em texto completo ou que não estavam na língua portuguesa.

Os artigos selecionados foram analisados através da leitura do texto completo, com marcação e seleção dos tópicos concernentes à questão norteadora. Os dados foram sintetizados e comparados com apontamentos estruturados para a obtenção dos resultados, e estes, apresentados no formato de quadro descritivo.

Conforme os descritores estabelecidos, foi possível obter nas duas bases de dados pesquisadas, 478 artigos na totalidade. Após a leitura criteriosa dos títulos e resumos das publicações, foram selecionados, três trabalhos da base de dados Scielo e dois da base de dados Medline. Desta forma, foram selecionados cinco artigos que tratavam de alterações morfológicas em cérebros de pessoas diagnosticadas com depressão.

\section{DESENVOLVIMENTO}


Os artigos incluídos estão descritos no Quadro 1.

\begin{tabular}{|c|c|c|c|c|}
\hline $\begin{array}{l}\text { Periódico / } \\
\text { Código }\end{array}$ & Autores/ano & Título & Metodologia & Resultados obtidos \\
\hline $\begin{array}{l}\text { Journal of } \\
\text { affective } \\
\text { disorder* }\end{array}$ & $\begin{array}{l}\text { Deckersbach } \\
\text { et al. (2018) }\end{array}$ & $\begin{array}{l}\text { O desempenho da } \\
\text { memória prediz } \\
\text { resposta para a } \\
\text { depressão no } \\
\text { tratamento bipolar: Um } \\
\text { piloto randomizado } \\
\text { controlados com } \\
\text { ressonância magnética }\end{array}$ & $\begin{array}{l}\text { Estudo clínico } \\
\text { randomizado }\end{array}$ & $\begin{array}{l}\text { A melhora no } \\
\text { tratamento da } \\
\text { depressão envolve } \\
\text { estruturas límbicas e } \\
\text { regiões frontal e } \\
\text { medial do cérebro }\end{array}$ \\
\hline $\begin{array}{l}\text { Translational } \\
\text { Psychiatry* } \\
\text { Artigo } 2\end{array}$ & $\begin{array}{l}\text { Renteria et } \\
\text { al. (2017) }\end{array}$ & $\begin{array}{l}\text { Estrutura cerebral } \\
\text { subcortical e } \\
\text { comportamento suicida } \\
\text { no transtorno } \\
\text { depressivo maior: Uma } \\
\text { meta-análise do grupo } \\
\text { de trabalho ENIGMA - } \\
\text { TDM }\end{array}$ & Meta-análise & $\begin{array}{l}\text { Pacientes com } \\
\text { depressão exibem } \\
\text { menores volumes } \\
\text { intracranianos e } \\
\text { subcorticais e } \\
\text { maiores volumes } \\
\text { ventriculares. }\end{array}$ \\
\hline $\begin{array}{l}\text { Revista } \\
\text { Brasileira de } \\
\text { Epidemiologia** } \\
\text { Artigo } 3\end{array}$ & $\begin{array}{c}\text { Stopa et al. } \\
(2015)\end{array}$ & $\begin{array}{l}\text { Prevalência do } \\
\text { autorelato de depressão } \\
\text { no Brasil: Resultados } \\
\text { da pesquisa nacional de } \\
\text { saúde, } 2013 \text {. }\end{array}$ & $\begin{array}{l}\text { Inquérito de } \\
\text { base } \\
\text { populacional }\end{array}$ & $\begin{array}{l}\text { O autorelato de } \\
\text { depressão varia por } \\
\text { escolaridade, raça, } \\
\text { faixa etária, local de } \\
\text { residência e região. }\end{array}$ \\
\hline $\begin{array}{l}\text { Enfermería: } \\
\text { Cuidados } \\
\text { humanizados** } \\
\text { Artigo } 4\end{array}$ & $\begin{array}{l}\text { Campos e } \\
\text { Feitosa } \\
(2017)\end{array}$ & $\begin{array}{l}\text { Elaboração de um } \\
\text { protocolo para o } \\
\text { diagnóstico da } \\
\text { depressão. }\end{array}$ & $\begin{array}{l}\text { Revisão não } \\
\text { sistemática da } \\
\text { literatura }\end{array}$ & $\begin{array}{l}\text { Construção de um } \\
\text { protocolo de } \\
\text { diagnóstico de } \\
\text { depressão. }\end{array}$ \\
\hline $\begin{array}{l}\text { Revista médica } \\
\text { uruguaia** } \\
\text { Artigo } 5\end{array}$ & $\begin{array}{l}\text { Castrillàn et } \\
\text { al. (2013) }\end{array}$ & $\begin{array}{l}\text { Gliomatosis cerebral: A } \\
\text { propósito de dois casos } \\
\text { clínicos }\end{array}$ & Estudo de caso & $\begin{array}{l}\text { Ressonância } \\
\text { magnética é um } \\
\text { método de eleição } \\
\text { para o diagnóstico de } \\
\text { alterações cerebrais. }\end{array}$ \\
\hline
\end{tabular}

Notas: *Base de dados Medline; ${ }^{* \star}$ Base de dados Scielo.

Fonte: os autores.

Estudos morfológicos, neuropsicológicos e neurobiológicos da depressão têm como foco principal correlacionar os estados clínico e patológico para se estabelecer maior entendimento do transtorno e consequentemente proporcionar maiores benefícios para o paciente deprimido.

O DSM IV caracteriza a depressão como um conjunto de transtornos, sendo sistematizados como transtornos de humor. De acordo com o CID 10, clinicamente a depressão é definida como a dificuldade para prosseguir com o trabalho e com as atividades sociais normais. É classificada por sua gravidade, em leve, moderada e grave, limitando-se a três sintomas fundamentais: humor deprimido ou tristeza persistente, fadigabilidade aumentada ou perda de energia física ou mental, e perda de interesse e prazer (OMS, 1998). Com o objetivo de elaborar um protocolo para diagnóstico da depressão por profissionais de saúde médicos e não médicos, Campos e Feitosa (2017), (Artigo 4), descrevem que no caso da depressão, quanto mais rápido for o diagnóstico, melhores são as chances da resposta ao tratamento ser positiva.

Para Gonçalves et al. (2018), a lacuna na utilização de instrumentos de 
rastreamento de depressão na atenção primária pode estar vinculada a não capacitação dos profissionais e/ou ao reduzido tempo das consultas. Os profissionais ainda estão focados especialmente na queixa do paciente e nem sempre consideram seu contexto de vida, enfatizando uma ação limitada e ineficaz dos serviços de saúde, que também não se articulam e se integram aos serviços especializados em saúde mental.

Em um inquérito de base populacional baseado nos resultados da Pesquisa Nacional em Saúde (PNS) em 2013 sobre a prevalência do autorrelato da depressão no Brasil, Stopa et al. (2015), (Artigo 3), mensuraram que 7,6\% dos entrevistados afirmaram ter recebido o diagnóstico médico prévio de depressão em algum momento da vida, sendo maior em mulheres (10,9\%), e em pessoas de 60 a 64 anos (11,1\%). As maiores prevalências foram encontradas em indivíduos sem instrução ou com ensino fundamental incompleto. Os autores afirmam ainda que segundo a PNS, $8,6 \%$ dos adultos referiram ter recebido, em algum momento da vida, diagnóstico prévio positivo de depressão feito por médico ou profissional de saúde mental.

Além do diagnóstico baseado em autorrelatos e aplicação de protocolos, os exames de imagem, como a ressonância magnética e a tomografia computadorizada, surgem como possibilidade de um meio diagnóstico mais preciso da depressão. Segundo Renteria et al. (2017), métodos de neuroimagem que proporcionam uma abordagem não invasiva para explorar as correlações neurais do comportamento mostram resultados promissores, e revelam alterações neuromorfológicas.

Estudo de meta análise conduzido por Renteria et al. (2017), (Artigo 2), teve por objetivo estudar as alterações morfofuncionais apresentadas em exames de ressonância magnéticas em pacientes diagnosticados com transtorno depressivo maior (TDM) e com tendências suicidas. O estudo comparou volumes médios subcorticais da substância cinzenta, volume do ventrículo lateral e volume intercraniano total de pacientes com sintomas de TDM com imagens das mesmas estruturas em indivíduos controles saudáveis. Dessa observação constatou-se que pessoas diagnosticadas com depressão apresentavam volumes subcoticais menores e volumes ventriculares maiores em comparação com os controles, além de menores volumes de substância cinzenta do córtex orbitofrontal. Para os autores, alterações cerebrais, mesmo em um pequeno tamanho, podem ter relevância clínica se estão associadas com a resposta ao tratamento. Afirmam também que, juntamente com o córtex cingulado anterior e córtex orbitofrontal, regiões subcorticais do estriado podem ser particularmente relevantes para estudos de vulnerabilidade do estado deprimido.

Em outra pesquisa que utilizou neuroimagens no diagnóstico de depressão por meio de um estudo clínico randomizado, Deckersbach et al. (2018), (Artigo 1), avaliaram 32 adultos com idade entre 18 e 65 anos. Utilizaram os critérios de depressão do DSM-IV para diagnóstico e analisaram 0 desempenho da memória exploratória verbal durante o exame de ressonância magnética. Os autores identificaram que estudos de neuroimagem mostram consistentes anormalidades do córtex pré-frontal e do lobo temporal, e que durante tratamentos psicoterápicos ocorriam aumento da ativação do córtex préfrontal e envolvimento das estruturas límbicas (hipocampo), concluindo que a 
melhora do paciente durante o tratamento está relacionada com grau de envolvimento dessas estruturas cerebrais.

Porto, Hermolin e Ventura (2002), elucidam, em seu estudo, que a avaliação de regiões específicas corticais pela ressonância magnética mostra evidência de alterações no lobo frontal de pacientes com depressão, e que há fortes evidências de diminuição de fluxo sanguíneo e metabolismo nessa região.

Castrillón, Leizagoen e El Herdoy (2013), (Artigo 5), analisou exames de TC de cérebros de pacientes acometidos por gliomatose cerebral, uma neoplasia primária, e sua relação com o estado deprimido desses pacientes. Constataram que as imagens apresentam anormalidade, com a perda de diferenciação entre a substância branca e cinzenta, o que sugere uma relação com a depressão. Afirmam ainda que exames de neuroimagens devem ser um método de eleição para o diagnóstico de depressão.

Pesquisa de Fujino et al. (2014) sobre estudos de neuroimagem em pacientes com TDM apontam que o córtex cingulado anterior é uma estrutura que tem sido repetidamente relatada com alterações em pacientes com TDM. Esta região está envolvida em várias funções cognitivas, tais como, a tomada de decisão, o controle da inibição, e a empatia. Os autores encontraram uma forte relação entre a parte caudal do córtex cingulado anterior com o volume de substância cinzenta nesta região, e sugerem que a análise desse volume pode prever respostas para o tratamento de pacientes com TDM.

Porto, Hermolin e Ventura (2002), esclarecem que os estudos neuropsicológicos com deprimidos apontam para alterações na aquisição da memória, atenção, concentração, flexibilidade cognitiva e abstração, e concluem que a depressão deve ser entendida e avaliada sob múltiplos aspectos, e daí a importância de uma equipe multidisciplinar visando uma melhor qualidade de tratamento.

Para Davidson et al. (2002), cada vez mais os estudos científicos desenvolvidos nesta área vêm considerando, além dos aspectos mentais propriamente ditos (como na psicologia cognitiva), os seus substratos neuroanatômicos e neurofuncionais (neuropsicologia, neurobiologia). Assim, a psiquiatria se insere no vasto campo das neurociências cognitivas, beneficiandose de instrumentos que explicam as disfunções cognitivas com base no conhecimento da relação cérebro e mente.

\section{CONCLUSÃO}

Após a análise dos artigos selecionados, conclui-se que o cérebro de pessoas diagnosticadas com depressão podem apresentam alterações morfológicas visíveis em exames de neuroimagens, sendo estas assim caracterizadas: volumes subcorticais menores, volumes ventriculares maiores, menor volume da substância cinzenta do córtex orbito-frontal, anormalidade no córtex pré-frontal, perda de diferenciação entre substância branca e cinzenta e modulações das estruturas límbicas no processo do quadro depressivo.

Desta maneira, parece evidente que exames de neuroimagens são um recurso importante para a obtenção de um diagnóstico mais preciso da depressão, bem como para reduzir o tempo de busca por achados clínicos, e consequentemente erros de diagnósticos e uso de fármacos inadequados. 
Neste sentido, este estudo sugere a necessidade de que outras pesquisas que avaliem a neuroimagem como forma de diagnóstico da depressão sejam realizadas. Esses achados podem contribuir, assim, para que estes exames sejam incluídos em protocolos de avaliação diagnóstica de pacientes com sintomas depressivos, e consequentemente, para que o tratamento seja iniciado de forma mais rápida e assertiva.

\section{REFERÊNCIAS}

CASTRILLÓN, C.; LEIZAGOEN, F.; EL HORDOY, F. Gliomatosis cerebral: a propósito de dos casos clínicos. Revista Médica del Uruguay, v. 29, n. 4, p. 237-241, 2013.

CAMPOS, A. A. C.; FEITOSA, F. B. Elaboração de um protocolo para o diagnóstico da depressão. Enfermeria: cuidados humanizados, v. 6, n. 2, p. 21-31, 2017.

COFFEY, C. E. et al. Leukoencephalopathy in elderly depressed patients referred for ECT. Biological Psychiatry, v. 24, n. 2, p. 143-161, 1998.

COOPER, H. M. Synthesizing research: a guide for literature reviews. 3. ed. Thousand Oaks, CA: Sage Publications, 1998.

DECKERSBACH, T. et al. Memory performance predicts response to psychotherapy for depression in bipolare disorder: A pilot randomized controlled trial with exploratory functional magnetic resonance imaging. Journal of Affective Disorders, v. 229, p. 342-350, 2018.

DAVIDSON, R. J. et al. Neural and behavioral substrates of mood and mood regulation. Biological Psychiatry, v. 52, n. 6, p. 478-502, 2002.

AMERICAN PSYCHIATRIC ASSOCIATION. DSM - IV. 4. ed. Washington, DC: American Psychiatric Associacion, 1994.

FUJINO, J. et al. O volume cingulado anterior prediz resposta a terapia cognitivo comportamental no transtorno depressivo maior. Jornal of Afective Disorders, v. 174, p. 397-399, 2014.

GONÇALVES, A. M. C. et al. Prevalência de depressão e fatores associados em mulheres atendidas pela Estratégia de Saúde da Família. Jornal Brasileiro de Psiquiatria, v. 67, n. 2, p. 101-109, 2018.

LOPES, C. S. et al. Inequities in access to depression treatment: results of the Brazilian National Health Survey - PNS. International Journal for Equity in Health, v. 15, n. 1, p. 1-21, 2016.

MENDES, K. D. S.; SILVEIRA, R. C. C. P.; GALVÃO, C. M. Revisão integrativa: método de pesquisa para incorporação de evidências na saúde e na 
enfermagem. Texto Contexto Enfermagem, v. 17, n. 4, p. 758-764, 2008.

ORGANIZAÇÃO MUNDIAL DA SAÚDE. Classificação dos transtornos mentais e de comportamento da CID-10: descrições clínicas e diretrizes diagnósticas. Porto Alegre: Artes médicas, 1998.

PORTO, P.; HERMOLIN, M.; VENTURA, P. Alterações neuropsicológicas associadas à depressão. Revista Brasileira de Terapia Comportamental e Cognitiva. v. 4, n. 1, p. 63-70, 2002.

RENTERIA, M. E. et al. Subcortical brain structure and suicidal behaviour in major depressive disorder: a meta-analysis from the ENIGMA-MDD working group. Translatinal Psychiatry, v. 7, e1116, 2017.

SANTOS, I. S. et al. Sensitivity and specificity of the Patient Health Questionnaire-9 (PHQ-9) among adults from the general population. Caderno de Saúde Pública, v. 29, n. 8, p. 1533-1543, 2013.

STOPA, S. R. et al. Prevalência do autorrelato de depressão no Brasil: resultados da pesquisa nacional de saúde, 2013. Revista Brasileira de Epidemiologia, v. 18, n. Suppl 2, p. 170-180, 2015.

WEIGH, G.; BIGLER, E. D. Neuroimaging in Psychiatric. The Psychiatric Clinics of North American, v. 21, n. 4, p. 725-759, 1998.

WORLD HEALTH ORGANIZATION. Depression and Other Common Mental Disorders - Global Health Estimates. Geneva: WHO/MSD/MER, 2017. 